\title{
S-(2-乙酰苯基)-二甲基硫代氨基甲酸酯环合产物的确定
}

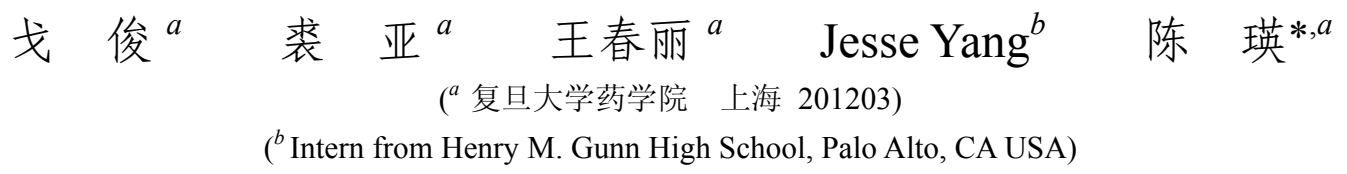

\begin{abstract}
摘要 通过 ${ }^{1} \mathrm{H} N M R, N O E$ 和 X-ray 等方法, 确定了 $S$-(2-乙酰苯基)-二甲基硫代氨基甲酸酯在 $\mathrm{NaH} / \mathrm{DMF}$ 条件下的环合 产物为苯并- $2 H$-噻喃-2-酮, 而非文献报道的苯并- $4 H$-璂喃-4-酮. 探讨了苯并- $2 H$-噻喃-2-酮和苯并- $4 H$-噻喃-4-酮结构在 氢谱上的规律性差异.

关链词＼cjkstart苯并-4H-噻喃-4-酮; 苯并- $2 H$-噻喃-2-酮; 环合; $S$-(2-乙酰苯基)-二甲基硫代氨基甲酸酯
\end{abstract}

\section{Clarification on the Structure of Cyclization Product of S-2-Acetylphenyl Dimethylthiocarbamate}

\author{
Ge, Jun ${ }^{a} \quad$ Qiu, Ya ${ }^{a} \quad$ Wang, Chunli ${ }^{a} \quad$ Yang, Jesse $^{b} \quad$ Chen, Ying ${ }^{*, a}$ \\ ( ${ }^{a}$ Department of Medicinal Chemistry, School of Pharmacy, Fudan University, Shanghai, China) \\ ( ${ }^{b}$ Intern from Henry M. Gunn High School, Palo Alto, CA USA)
}

\begin{abstract}
The structure of cyclization product from the reaction of $S$-2-acetylphenyl dimethylthiocarbamate with NaH in DMF was clarified by ${ }^{1} \mathrm{H}$ NMR, NOE and X-ray. It proved to be a benzo- $2 H$-thiopyran-2-one instead of a benzo- $4 H$ thiopyran-4-one reported in literature. The ${ }^{1} \mathrm{H}$ MNR chemical shift data of seven benzo- $2 H$-thiopyran-2-ones and benzo- $4 H$ thiopyran-4-ones were discussed, which might be useful for distinguishing benzo- $H$-thiopyran-2-ones from benzo- $4 H$ thiopyran-4-ones.
\end{abstract}

Keywords benzo- $4 H$-thiopyran-4-one; benzo- $2 H$-thiopyran-2-one; cyclization; $S$-2-acetylphenyl dimethylthiocarbamate

Suksdorfin (1) 是从植物 Lomatium Suksdorfii ${ }^{[1]}$ 的果 实中分离得到的一种天然产物, 对其进行结构修饰得到 具有显著抗 HIV 活性的 4-甲基-DCK [3',4'-di-O-(S)-camphanoyl-(+)-cis-khellactone] (2) ${ }^{[2]}$ 和 2-乙基-DCP $\left\{3^{\prime} R\right.$, 4'R-di- $O$-(S)-camphanoyl-2',2'-dimethyldihydropyrano[2, 3-f]chromone\} (3) ${ }^{[3]}$. 我们之前的研究表明, 一些 1-thia-DCKs (4) ${ }^{[4]}$ 显示了比 DCK 更好的抗 HIV 活性. 对 DCK 类化合物结构简化修饰得到一系列 C 环开环的 DCKs (seco-DCK 5) ${ }^{[5]}$ 和 DCPs (seco-DCP 6) ${ }^{[6]}$, 它们不 但具有比化合物 2 更好的化学稳定性和抗 HIV 活性, 还 对多药耐药的 HIV-1 病毒株显示出良好的敏感性. 因此 我们进一步设计了一系列新的 seco-DCKs 和 seco-DCPs 的生物电子等排体, 包括 1-硫杂的 seco-DCK (7)和 seco-DCP $(\mathbf{8})$ 化合物, 考察其活性.
Lau 等 ${ }^{[7]}$ 报道了 $S$-(2-乙酰苯基)-二甲基硫代氨基甲 酸酯在 DMF/ $\mathrm{NaH}$ 条件下发生的环合反应, 其产物苯并$4 H$-噻喃-4-酮(9)可以作为合成目标化合物 8 的中间体. 但是这篇文献的数据并不能排除环合产物是苯并 $-2 H$ 噻喃-2-酮(10)结构的可能性.

我们重复该文献的环合反应, 对产物进行了结构确 证研究, 阐明了所得的环合产物为苯并- $2 H$-噻喃-2-酮 (10)结构, 并非文献报道的 4-酮化合物 9 的结构骨架. 同时, 通过高斯软件计算 ${ }^{[8]}, 9 \mathrm{a}$ 的能量为 -6716.810 $\mathrm{J} / \mathrm{mol}, 10 \mathrm{a}$ 的能量为 $-6716.881 \mathrm{~J} / \mathrm{mol}$, 结果显示它们并 没有显著能量差异. 另外, 我们以 $\mathbf{1 0}$ 为中间体合成了目 标化合物 7; 并进一步通过环合产物的烷基化反应，讨 论了苯并- $2 H$-噻喃-2-酮(10)和苯并- $4 H$-噻喃-4-酮(9)两 类化合物氢谱化学位移的规律性差异.

\footnotetext{
* E-mail: yingchen71@fudan.edu.cn

Received April 11, 2012; revised July 24, 2012; published online August 6, 2012.

Project supported by the National Natural Science Foundation of China (Nos. 20272010, 30200348, 30873164).

国家自然科学基金(Nos. 20272010, 30200348, 30873164)资助项目.
} 


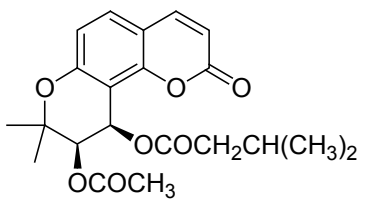

Suksdorfin (1)

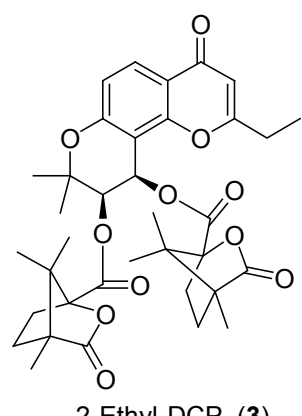

2-Ethyl-DCP (3)<smiles>CCc1cc(=O)c2ccc(OC(C)C)c(COC(=O)C34CCC(C)(C(=O)O3)C4(C)C)c2o1</smiles>

5<smiles>[3H]C12CCC(C(=O)OCc3c(OC(C)C)ccc4c(OC)cc(=O)sc34)(OC1=O)C2(C)C</smiles><smiles>[X]c1ccc(C(C)=O)c(SC(=O)N(C)C)c1</smiles>

S-2-acetylphenyl dimethylthiocarbamate<smiles>[X]c1ccc2sc(=O)cc(O)c2c1</smiles>

10<smiles>COc1ccc2c(=O)cc(O)sc2c1</smiles><smiles>COc1ccc2c(O)cc(=O)sc2c1</smiles>

$10 \mathrm{a}$

\section{1 结果与讨论}

如 Scheme 1 所示, 以化合物 11 为原料合成得到中 间体 $S$-(2-乙酰苯基)-二甲基硫代氨基甲酸酯(13), 并参
照 $\mathrm{Lau}$ 的方法分别由 13a 和 $13 \mathrm{~b}$ 环合得到 10a 和 $10 b$.<smiles>[R]Oc1ccc(O)c(C(C)=O)c1</smiles>

a: $\mathrm{R}=\mathrm{CH}_{3}$

b: $\mathrm{R}=\mathrm{CH}_{2} \mathrm{OCH}_{3}$

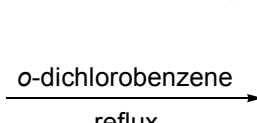

reflux
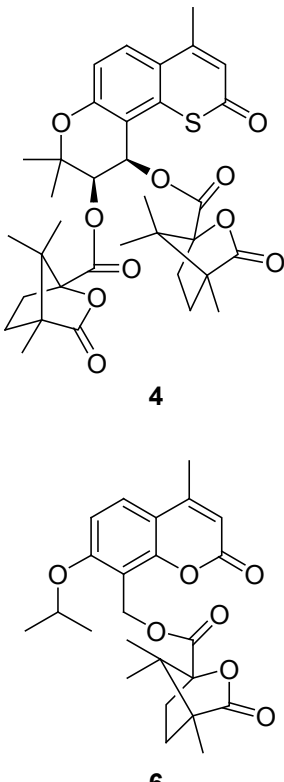<smiles>COc1cc(=O)c2ccc(OC(C)C)c(COC(=O)C3(C)C(=O)OC(C)(C)C3(C)C)c2o1</smiles><smiles>[X]c1ccc2sc(O)cc(=O)c2c1</smiles>

9

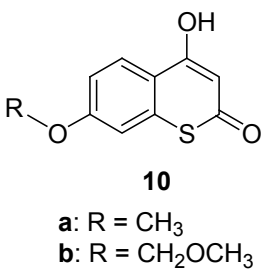

dimethylthiocarbamoyl chloride

$\mathrm{NaH}, \mathrm{DMF}$<smiles>[R]Oc1ccc(C(C)=O)c(OC(=S)N(C)C)c1</smiles>

(1) $\mathrm{NaH}, \mathrm{DMF}$

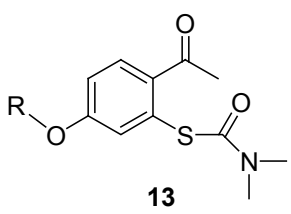

a: $\mathrm{R}=\mathrm{CH}_{3}$ b: $\mathrm{R}=\mathrm{CH}_{2} \mathrm{OCH}_{3}$ 12 a: $\mathrm{R}=\mathrm{CH}_{3}$ b: $\mathrm{R}=\mathrm{CH}_{2} \mathrm{OCH}_{3}$

(2) $1 \mathrm{~mol} / \mathrm{L} \mathrm{HCl}$

\section{Scheme 1}

由于该文献没有提供环合产物的氢谱数据，我们所 合成相应化合物无论是苯并- $4 H$-噻喃-4-酮(9)还是苯并$2 H$-噻喃-2-酮(10)结构都没有直接对照. 因此，我们用 与文献报道一致的底物(13a)和反应条件合成得到 10a, 测定了其 $\mathrm{X}$ 单晶衍射结构(图 1), 晶体结构显示噻喃上 的羰基位于 2-位，而非 4-位.

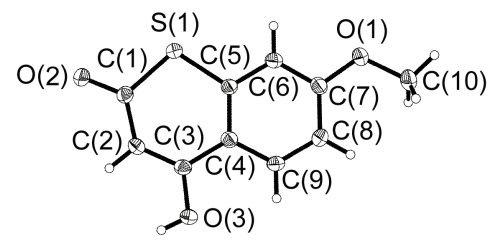

图 $110 a$ 的单晶衍射结构

Figure 1 Crystal structure of 10a

化合物 $10 \mathrm{~b}$ 的单晶没能培养成功, 我们测定了其 $\mathrm{NOE}$ 和 $\mathrm{HMBC}$ 谱以确定其结构. 在其 $\mathrm{NOE}$ 谱中, 当辐 射着基氢 $(\delta 12.01)$ 时可明显观测到 3-H $(\delta$ 5.93)与 5- $\mathrm{H}(\delta$ 8.04 ) 存在相关性. 而其 HMBC 谱则显示 5-H ( ${ }^{1} \mathrm{H}$ NMR $\delta$ $8.04)$ 和羰基碳 $\left({ }^{13} \mathrm{C}\right.$ NMR $\delta$ 181.92)无明显相关. 这些数 据与化合物 $10 \mathrm{~b}$ 为苯并- $2 H$-噻喃-2-酮的结构特征相吻 合.

而后，我们进一步以化合物 10b 为原料经 Scheme 2 路线合成了目标产物 7 , 且顺利得到了单晶并测定其 $\mathrm{X}$ 衍射结构(图 2), 同样显示羰基处在噻喃酮的 2-位, 进一 步间接证实了化合物 $10 \mathbf{b}$ 为苯并 $-2 H$-噻喃-2-酮的结构.

此外，以化合物 $10 \mathrm{~b}$ 为原料，在 $\mathrm{K}_{2} \mathrm{CO}_{3} / \mathrm{DMF}$ 条件下 与卤代烷发生烷基化可生成少量互变异构的苯并- $4 H$ 噻喃-4-酮结构的产物 $(\mathbf{2 7}, \mathbf{2 9})$ (Scheme 3), 揭示了苯并- 


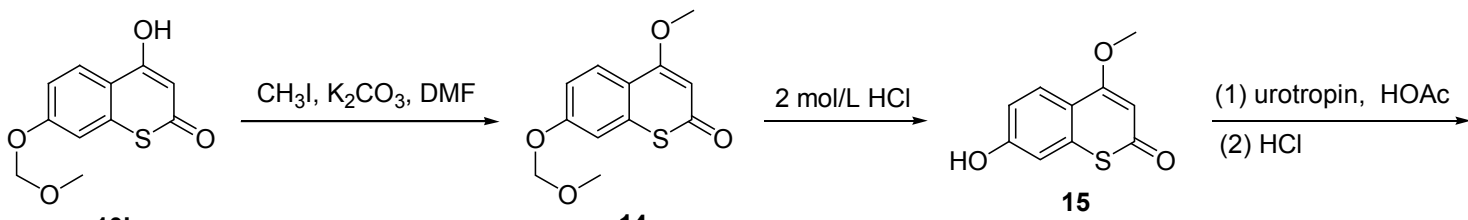

$10 \mathrm{~b}$<smiles>COc1cc(=O)sc2c(C3OCCO3)c(O)ccc12</smiles>

17<smiles>COc1cc(=O)sc2c(C3OCCO3)c(OC(C)C)ccc12</smiles>

18

16<smiles>COc1cc(=O)sc2c(CO)c(OC(C)C)ccc12</smiles>

camphanoyl chloride, DMAP, $\mathrm{CH}_{2} \mathrm{Cl}_{2}$<smiles>COc1cc(=O)sc2c(OC(=O)C3(C)CCC(C)(C)C(=O)O3)c(OC(C)C)ccc12</smiles>

Scheme 2

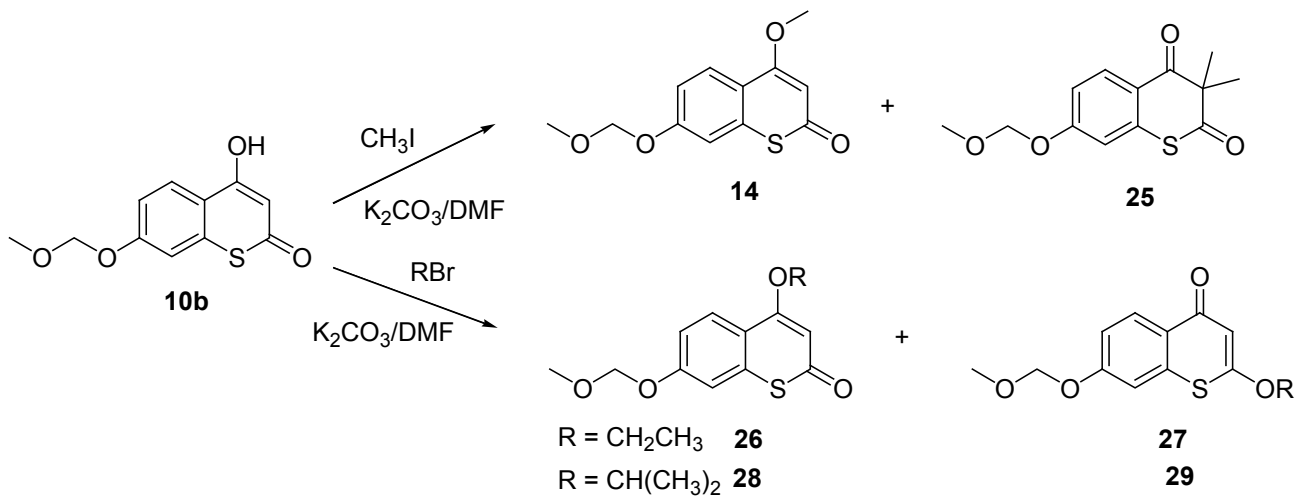

Scheme 3

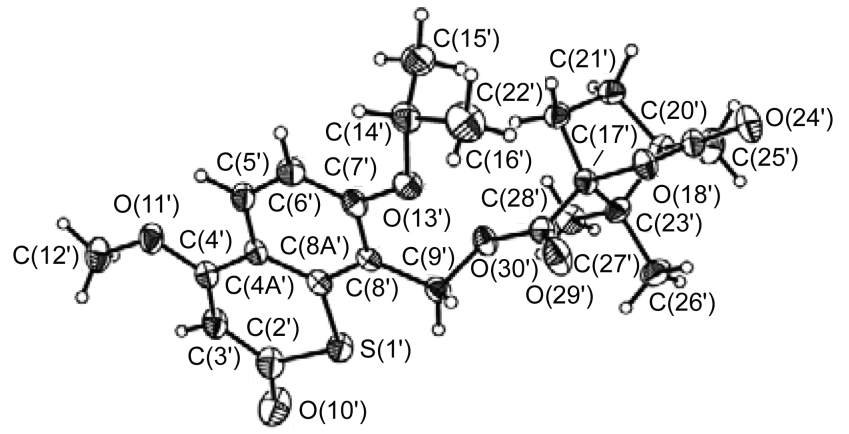

图 27 的单晶衍射结构

Figure 2 Crystal structure of 7

$2 \mathrm{H}$-噻喃-2-酮在适当的反应条件下即可转化生成苯并4H-噻喃-4-酮异构体. 这暗示此两种异构体可以通过选 择合适的碱性试剂和反应溶剂来控制互变异构平衡以 获得相应产物.

表 1 列出化合物 $\mathbf{1 0 a}, \mathbf{1 0 b}$ 和烷基化产物的 ${ }^{1} \mathrm{H}$ MNR
化学位移数据, 可以发现本研究中所得苯并- $2 H$-噻喃-2酮化合物中的 $3-\mathrm{H}$ 和 $5-\mathrm{H}$ 化学位移值普遍比苯并 $-4 H-$ 噻喃-4-酮结构中的相应 $\delta$ 值小约 0.3. 这可能是在苯并4H-噻喃-4-酮骨架中与苯环相连的酮基对近位的 5- $\mathrm{H}$ 和 邻位的 3-H 产生更明显的去屏蔽效应，使 $\delta$ 值向低场移 动. 这种具有规律性的化学位移上的差别对于区别这两 种异构体的结构有一定的参考意义.

\section{2 结论}

在合成关键中间体 $10 \mathrm{~b}$ 的过程中发现文献[7]报道 的 $S$-(2-乙酰苯基)-二甲基硫代氨基甲酸酯的环合产物 的结构判断数据不充分, 本文综合 ${ }^{1} \mathrm{H} \mathrm{NMR,NOE}$ 数据 和单晶结构信息, 确定了在文献反应体系中的环合产物 应为苯并- $2 H$-噻喃-2-酮结构, 而非 4-酮结构; 同时还总 结了本研究中所得苯并- $2 H$-噻喃-2-酮结构和苯并- $4 H$ 噻喃-4-酮结构化合物的氢谱化学位移的规律性差异. 
表 1 部分苯并- $2 H$-噻喃-2-酮和苯并- $4 H$-噻喃-4-酮的氢谱化 学位移值

Table $1{ }^{1} \mathrm{H}$ MNR chemical shift data of some benzo- $2 H$-thiopyran-2-ones and benzo-4H-thiopyran-4-ones

\begin{tabular}{ccccc}
\hline Compd. & $\delta(3-\mathrm{H})$ & $\delta(5-\mathrm{H})$ & $\delta(6-\mathrm{H})$ & $\delta(8-\mathrm{H})$ \\
\hline $\mathbf{1 0 a}$ & 5.92 & 8.02 & 7.03 & 7.16 \\
$\mathbf{1 0 b}$ & 5.93 & 8.04 & 7.10 & 7.19 \\
$\mathbf{1 4}$ & 5.99 & 8.05 & 7.04 & 7.10 \\
$\mathbf{2 6}$ & 5.98 & 8.08 & 7.04 & 7.09 \\
$\mathbf{2 8}$ & 5.97 & 8.06 & 7.03 & 7.09 \\
$\mathbf{2 7}$ & 6.30 & 8.38 & 7.12 & 7.11 \\
$\mathbf{2 9}$ & 6.31 & 8.38 & 7.11 & 7.10 \\
\hline
\end{tabular}

\section{3 实验部分}

\section{1 仪器与试剂}

熔点用毛细管法测定, 温度计未校正. TLC 用板为 $0.2 \mathrm{~mm}$ GF245 高效薄层层析硅胶板. 柱层析使用 300 400 目硅胶或硅胶 H 装柱. ${ }^{1} \mathrm{H}$ NMR 谱用 Bruker-DPX 400 $\mathrm{MHz}$ 或 Bruker-Advance $500 \mathrm{MHz}$ 核磁共振仪测定, 内 标为 TMS $(\delta 0.0)$ 或 $\mathrm{CDCl}_{3}\left(\delta\right.$ 7.27). ${ }^{13} \mathrm{C}$ NMR 谱在 Varian Mercury 300 核振共振仪上测定. MS 在 HP5973N 型质谱 仪上测定. $X$ 衍射由复旦大学分析测试中心测定. 所用 试剂均为市售分析纯或化学纯.

\section{2 实验方法}

2-二甲基硫代氨甲酰氧基-4-甲氧甲氧基-苯乙酩 (12b): 在 $50 \mathrm{~mL}$ 圆底瓶中, 加入化合物 $\mathbf{1 1 b}(1.0 \mathrm{~g}, 5.1$ $\mathrm{mmol}$ ), 碳酸钾 $(2.82 \mathrm{~g}, 20.4 \mathrm{mmol}), \mathrm{N}, \mathrm{N}$-二甲基硫代氨 甲酰氯(1.26 g, $10.2 \mathrm{mmol})$ 和甲醇约 $10 \mathrm{~mL}$, 室温搅拌反 应; $1 \mathrm{~h}$ 后原料基本消失完全; 反应液用约 $200 \mathrm{~mL}$ 冰水 水析, 抽滤, 得浅黄色固体, 乙酸乙酯重结晶得白色固 体 12b (1.3 g, 93.2\%). m.p. $118 \sim 119{ }^{\circ} \mathrm{C} ;{ }^{1} \mathrm{H}$ NMR $\left(\mathrm{CDCl}_{3}, 500 \mathrm{MHz}\right) \delta: 2.50\left(\mathrm{~s}, 3 \mathrm{H}, \mathrm{COCH}_{3}\right), 3.40(\mathrm{~s}, 3 \mathrm{H}$, $\left.\mathrm{OCH}_{3}\right), 3.47$ [d, $\left.J=10.25 \mathrm{~Hz}, 6 \mathrm{H}, \mathrm{N}\left(\mathrm{CH}_{3}\right)_{2}\right], 5.22(\mathrm{~s}, 2 \mathrm{H}$, $\left.\mathrm{OCH}_{2} \mathrm{O}\right), 6.76(\mathrm{~d}, J=2.41 \mathrm{~Hz}, 1 \mathrm{H}, 6-\mathrm{H}), 6.96(\mathrm{dd}, J=$ 8.73, $2.41 \mathrm{~Hz}, 1 \mathrm{H}, 4-\mathrm{H}), 7.80$ (d, $J=8.73 \mathrm{~Hz}, 1 \mathrm{H}, 3-\mathrm{H})$; MS (EI) $m / z(\%): 283\left(\mathrm{M}^{+}, 1.74\right), 88$ (100).

2-二甲基氨基甲酰硫基-4-甲氧甲氧基-苯乙酮 (13b): 化合物 $\mathbf{1 2 b}(9 \mathrm{~g}, 31.76 \mathrm{mmol})$ 置于 $100 \mathrm{~mL}$ 圆底瓶 中, 加入 1,2 -二氯苯约 $38 \mathrm{~mL}$, 氮气保护下加热回流反 应; $9 \mathrm{~h}$ 后停止反应, 冷却后反应液直接湿法上硅胶柱层 析分离 [洗脱剂: $V$ (石油醚) $: V$ (乙酸乙酯 $)=8: 1$ ] 得主产 物点黄色油状物 13b (3.4 g, 34.4\%). ${ }^{1} \mathrm{H}$ NMR $\left(\mathrm{CDCl}_{3}\right.$, $400 \mathrm{MHz}) \delta$ : $2.57\left(\mathrm{~s}, 3 \mathrm{H}, \mathrm{COCH}_{3}\right), 3.07[\mathrm{br} \mathrm{s}, 6 \mathrm{H}$, $\left.\mathrm{N}\left(\mathrm{CH}_{3}\right)_{2}\right], 3.47$ (s, 3H, OCH 3$), 5.21\left(\mathrm{~s}, 2 \mathrm{H}, \mathrm{OCH}_{2} \mathrm{O}\right), 7.07$ (dd, $J=8.61,2.41 \mathrm{~Hz}, 1 \mathrm{H}, 4-\mathrm{H}), 7.28$ (d, $J=2.41 \mathrm{~Hz}, 1 \mathrm{H}$,
6-H), 7.65 (d, $J=8.61 \mathrm{~Hz}, 1 \mathrm{H}, 3-\mathrm{H})$; MS (EI) $m / z(\%): 283$ $\left(\mathrm{M}^{+}, 2.36\right), 72(100)$.

1-硫杂-4-羟基-7-甲氧甲氧基- $2 H$-色烯-2-酮(10b): 化合物 13b (1.79 g, $6.32 \mathrm{mmol})$ 加入约 $22 \mathrm{~mL}$ 无水 DMF 溶解, 加入 $\mathrm{NaH}$ 固体 $(365 \mathrm{mg}, 7.6 \mathrm{mmol}, 50 \%$ in mineral oil), 氮气保护, 室温条件下搅拌反应; $9 \mathrm{~h}$ 后原料完全消 失, 用 $1 \mathrm{~mol} / \mathrm{L} \mathrm{HCl}$ (约 $30 \mathrm{~mL}$ ) 调节反应液至中性, 乙酸 乙酯萃取, 合并有机相, 干燥、过滤, 浓缩得红棕色油状 物, 经硅胶柱层析分离[洗脱剂: $V$ (石油醚) $: V$ (乙酸乙 酯) $=4:$ ] 得化合物 10b (1.15 g, 76.8\%). m.p. 168 169 ${ }^{\circ} \mathrm{C}$; ${ }^{1} \mathrm{H}$ NMR (DMSO- $\left.d_{6}, 400 \mathrm{~Hz}\right) \delta: 3.37\left(\mathrm{~s}, 3 \mathrm{H}, \mathrm{OCH}_{3}\right)$, $5.28\left(\mathrm{~s}, 2 \mathrm{H}, \mathrm{OCH}_{2} \mathrm{O}\right), 5.93(\mathrm{~s}, 1 \mathrm{H}, 3-\mathrm{H}), 7.10$ (dd, $J=8.99$, $2.35 \mathrm{~Hz}, 1 \mathrm{H}, 6-\mathrm{H}), 7.19$ (d, $J=2.35 \mathrm{~Hz}, 1 \mathrm{H}, 8-\mathrm{H}), 8.04$ (d, $J=8.61 \mathrm{~Hz}, 1 \mathrm{H}, 5-\mathrm{H}), 12.10(\mathrm{~s}, 1 \mathrm{H}, \mathrm{OH})$; MS (EI) $\mathrm{m} / \mathrm{z}$ (\%): $238\left(\mathrm{M}^{+}, 14.87\right), 45$ (100).

1-硫杂-4-羟基-7-甲氧基- $2 H$-色烯-2-酮(10a): 化合 物 13a (0.25 g, $1 \mathrm{mmol})$ 加入无水 DMF $(10 \mathrm{~mL})$ 溶解, 加 入 $\mathrm{NaH}$ 固体 $(58 \mathrm{mg}, 1.2 \mathrm{mmol}, 50 \%$ in mineral oil), 氮气 保护，室温条件下摚拌反应; $9 \mathrm{~h}$ 后原料完全消失，用 1 $\mathrm{mol} / \mathrm{L} \mathrm{HCl}(30 \mathrm{~mL})$ 调节反应液至中性, 乙酸乙酯萃取, 合并有机相, 干燥、过滤, 浓缩得红棕色油状物, 经硅胶 柱层析分离[洗脱剂: $V$ (石油醚) $: V$ (乙酸乙酯 $)=4: 1$ ] 得 化合物 10a (129 mg, 62.0\%). m.p. 251 254 ${ }^{\circ} \mathrm{C} ;{ }^{1} \mathrm{H}$ NMR (DMSO- $\left.d_{6}, 400 \mathrm{~Hz}\right) \delta: 3.86\left(\mathrm{~s}, 3 \mathrm{H}, \mathrm{OCH}_{3}\right), 5.94(\mathrm{~s}, 1 \mathrm{H}$, 3-H), 7.06 (dd, $J=8.80,2.40 \mathrm{~Hz}, 1 \mathrm{H}, 6-\mathrm{H}), 7.19$ (d, $J=$ $2.00 \mathrm{~Hz}, 1 \mathrm{H}, 8-\mathrm{H}), 8.05$ (d, $J=9.20 \mathrm{~Hz}, 1 \mathrm{H}, 5-\mathrm{H}), 12.12$ (s, $1 \mathrm{H}, \mathrm{OH})$; HRMS calcd for $\mathrm{C}_{10} \mathrm{H}_{8} \mathrm{O}_{3} \mathrm{~S}\left(\mathrm{M}^{+}+\mathrm{H}\right)$ 209.0272, found 209.0266 .

1-硫杂-4-甲氧基-7-羊基- $2 \mathrm{H}$-色烯-2-酮(15): 化合物 $14(1.0 \mathrm{~g}, 3.97 \mathrm{mmol})$ 溶解于乙醇 $(35 \mathrm{~mL})$ 中, 加入 0.7 $\mathrm{mL} 2 \mathrm{~mol} / \mathrm{L}$ 盐酸; 加热回流反应, 反应液为浅红色, 0.5 $\mathrm{h}$ 后原料消失完全, 冷却, 减压蒸除溶剂, 过滤, 得浅粉 红色固体 15, 干燥约重 $800 \mathrm{mg}$, 收率 $97.0 \%$, 乙酸乙酯 重结晶得浅粉红色晶体. mp $213 \sim 214{ }^{\circ} \mathrm{C} ;{ }^{1} \mathrm{H}$ NMR (DMSO- $\left.d_{6}, 400 \mathrm{~Hz}\right) \delta: 3.95\left(\mathrm{~s}, 3 \mathrm{H}, \mathrm{OCH}_{3}\right), 6.00(\mathrm{~s}, 1 \mathrm{H}$, 3-H), 6.88 6.90 (m, 2H, 6-H and 8-H), 7.94 (d, $J=9.39$ $\mathrm{Hz}, 1 \mathrm{H}, 5-\mathrm{H}), 10.59$ (s, 1H, 7-OH); MS (ESI) $\mathrm{m} / z$ (\%): $209.1\left(\mathrm{M}^{+}+\mathrm{H}\right)$.

1-硫杂-4-甲氧基-7-羟基-8-甲酰基- $2 \mathrm{H}$-色烯-2-酮 (16): 化合物 15 (635 mg, $3 \mathrm{mmol})$ 和鸟洛托品 $(2.93 \mathrm{~g}, 21$ $\mathrm{mmol}$ )置于 $100 \mathrm{~mL}$ 圆底瓶中, 加入约 $15 \mathrm{~mL}$ 冰醋酸, 于 外温 $100{ }^{\circ} \mathrm{C}$ 左右加热反应; $1 \mathrm{~h}$ 后原料反应完全, 减压蒸 除约 $4 \mathrm{~mL}$ 冰醋酸后, 加入约 $11.3 \mathrm{~mL} 5 \mathrm{~mol} / \mathrm{L}$ 盐酸, 析 出较多浅黄色固体; 加热回流 $20 \mathrm{~min}$ 后, 反应液倾入约 $200 \mathrm{~mL}$ 冰水中, 析出大量浅黄色固体; 过滤, 固体干燥 
后用乙酸乙酯重结晶, 得浅黄色固体 16 (325 mg, $45.8 \%$ ). m.p. $264 \sim 265{ }^{\circ} \mathrm{C} ;{ }^{1} \mathrm{H}$ NMR (DMSO- $d_{6}, 400 \mathrm{~Hz}$ ) $\delta: 3.95$ (s, 3H, $\left.\mathrm{OCH}_{3}\right), 6.09$ (s, 1H, 3-H), 7.10 (d, $J=9.0$ $\mathrm{Hz}, 1 \mathrm{H}, 6-\mathrm{H}), 8.25$ (d, $J=9.0 \mathrm{~Hz}, 1 \mathrm{H}, 5-\mathrm{H}), 10.49(\mathrm{~s}, 1 \mathrm{H}$, 7-OH), 11.93 (s, 1H, 8-CHO); MS (EI) $m / z(\%): 236\left(\mathrm{M}^{+}\right.$, 45.38), 208 (100).

1-硫杂-8-(1,3-二氧杂戊烷-2-基)-7-差基-4-甲氧基$2 H$-苯并吡喃-2-酮(17): 化合物 16 (230 mg, $0.97 \mathrm{mmol})$, 乙二醇(182 mg, $2.92 \mathrm{mmol}$ )和 pTS 固体 $(10 \mathrm{mg}$, cat.)置于 $50 \mathrm{~mL}$ 圆底瓶中, 加入 $10 \mathrm{~mL}$ 溶剂苯, 回流分水反应; 2 $\mathrm{h}$ 后原料完全消失. 反应液稀释后用饱和碳酸氢钠水溶 液洗、饱和食盐水洗, 无水硫酸钠干燥, 过滤、浓缩析 出白色固体, 过滤得固体 $\mathbf{1 7}$ (220 mg, 81.5\%), 直接用于 下一步反应.

1-硫杂-8-(1,3-二氧杂戊烷-2-基)-7-异丙氧基-4-甲氧 基- $2 \mathrm{H}$-苯并吡喃-2-酮(18): 化合物 17 (180 mg, 0.64 $\mathrm{mmol}$ )溶于约 $10 \mathrm{~mL}$ 丙酮中, 加入无水碳酸钾 $(442 \mathrm{mg}$, $3.2 \mathrm{mmol})$, 硔化钾 $(20 \mathrm{mg}$, cat.) 和溴代异丙烷 $(0.2 \mathrm{~mL}$, $1.92 \mathrm{mmol}$ ), 加热回流反应, $5 \mathrm{~h}$ 后反应完; 溶剂浓缩后, 残留物用二氯甲烷溶解, 过滤去除不溶固体, 滤液浓缩 即析出白色固体 18 (160 mg, 77.3\%). m.p. 139 $140{ }^{\circ} \mathrm{C}$; ${ }^{1} \mathrm{H}$ NMR $\left(\mathrm{CDCl}_{3}, 400 \mathrm{~Hz}\right) \delta: 1.38(\mathrm{~d}, J=5.9 \mathrm{~Hz}, 6 \mathrm{H}, 2 \times$ $\mathrm{CH}_{3}$ in isopropyl), 3.95 (s, $\left.3 \mathrm{H}, \mathrm{OCH}_{3}\right), 4.04 \sim 4.38[\mathrm{~m}, 4 \mathrm{H}$, $\mathrm{O}\left(\mathrm{CH}_{2}\right)_{2} \mathrm{O}$ ], 4.66 4.72 (m, 1H, CH in isopropyl), 5.99 (s, 1H, 3-H), 6.48 (s, 1H, 9-H), 6.97 (d, $J=9.27 \mathrm{~Hz}, 1 \mathrm{H}, 6-\mathrm{H})$, 8.15 (d, $J=9.27 \mathrm{~Hz}, 1 \mathrm{H}, 5-\mathrm{H}$ ); MS (ESI) $m / z$ (\%): 323.2 $\left(\mathrm{M}^{+}+\mathrm{H}\right)$.

1-硫杂-4-甲氧基-7-异丙氧基-8-甲酰基- $2 \mathrm{H}$-色烯-2酮(19): 化合物 18 (150 mg, $0.47 \mathrm{mmol}$ )溶于约 $4.5 \mathrm{~mL}$ THF 溶剂中, 加入约 $3.5 \mathrm{~mL} 2 \mathrm{~mol} / \mathrm{L}$ 盐酸, 室温条件下 搅拌反应; $10 \mathrm{~min}$ 后原料完全消失, 反应液用乙酸乙酯 萃取多次; 有机相水洗至中性, 饱和食盐水洗, 无水硫 酸钠干燥、过滤、浓缩, 析出白色固体 19 (125 mg, 96.9\%). m.p. $212 \sim 214{ }^{\circ} \mathrm{C} ;{ }^{1} \mathrm{H}$ NMR $\left(\mathrm{CDCl}_{3}, 400 \mathrm{~Hz}\right) \delta$ : $1.46\left(\mathrm{~d}, J=5.9 \mathrm{~Hz}, 6 \mathrm{H}, 2 \times \mathrm{CH}_{3}\right.$ in isopropyl), $3.96(\mathrm{~s}, 3 \mathrm{H}$, $\left.\mathrm{OCH}_{3}\right), 4.80 \sim 4.86(\mathrm{~m}, 1 \mathrm{H}, \mathrm{CH}$ in isopropyl), $6.03(\mathrm{~s}, 1 \mathrm{H}$, 3-H), 7.04 (d, $J=9.0 \mathrm{~Hz}, 1 \mathrm{H}, 6-\mathrm{H}), 8.35$ (d, $J=9.0 \mathrm{~Hz}$, 1H, 5-H), 11.32 (s, 1H, 8-CHO); MS (EI) $m / z$ (\%): 278 $\left(\mathrm{M}^{+}, 28.60\right)$.

1-硫杂-4-甲氧基-7-异丙氧基-8-羟甲基- $2 \mathrm{H}$-色烯-2酮(20): 化合物 19 (90 mg, $0.32 \mathrm{mmol}$ )置于 $50 \mathrm{~mL}$ 圆底瓶 中, 加入约 $3.5 \mathrm{~mL}$ 甲醇, 搅拌未能溶解, 加入 $\mathrm{NaBH}_{4}$ (20 mg, $0.49 \mathrm{mmol})$, 轻微放热, 且原料完全溶解, 反应 液呈无色透明; $0.5 \mathrm{~h}$ 后原料反应完, 用 $2 \mathrm{~mol} / \mathrm{L}$ 盐酸调 节反应液至 $\mathrm{pH}$ 约为 $3 \sim 4$ 后, 用乙酸乙酯萃取, 有机相
水洗至中性, 饱和食盐水洗, 无水硫酸钠干燥, 过滤、浓 缩, 析出浅黄色固体, 过滤得化合物 $\mathbf{2 0}$ (88 $\mathrm{mg}, 97.1 \%)$. m.p. $122 \sim 124{ }^{\circ} \mathrm{C} ;{ }^{1} \mathrm{H}$ NMR $\left(\mathrm{CDCl}_{3}, 400 \mathrm{~Hz}\right) \delta: 1.42(\mathrm{~d}$, $J=6.3 \mathrm{~Hz}, 6 \mathrm{H}, 2 \times \mathrm{CH}_{3}$ in isopropyl), $2.28(\mathrm{br} \mathrm{s}, 1 \mathrm{H}, \mathrm{OH}$ ), $3.96\left(\mathrm{~s}, 3 \mathrm{H}, \mathrm{OCH}_{3}\right), 4.72 \sim 4.76(\mathrm{~m}, 1 \mathrm{H}, \mathrm{CH}$ in isopropyl), $4.86\left(\mathrm{~d}, J=5.1 \mathrm{~Hz}, 2 \mathrm{H}, \mathrm{CH}_{2} \mathrm{O}\right), 5.98(\mathrm{~s}, 1 \mathrm{H}, 3-\mathrm{H}), 6.98(\mathrm{~d}$, $J=9.0 \mathrm{~Hz}, 1 \mathrm{H}, 6-\mathrm{H}), 8.10(\mathrm{~d}, J=9.0 \mathrm{~Hz}, 1 \mathrm{H}, 5-\mathrm{H})$; MS (ESI) $m / z(\%): 281.0\left(\mathrm{M}^{+}+\mathrm{H}\right), 583.2\left(2 \mathrm{M}^{+}+\mathrm{Na}\right)$.

1-硫杂-4-甲氧基-7-异丙氧基-8-樟脑酯基亚甲基$2 H$-色烯-2-酩(7): 化合物 $\mathbf{2 0}$ (55 mg, $0.20 \mathrm{mmol}$ ), DMAP 固体 $(50 \mathrm{mg}, 0.40 \mathrm{mmol}$ )和 $(S)$-樟脑酰氯固体 $(65 \mathrm{mg}, 0.30$ $\mathrm{mmol}$ )置于 $50 \mathrm{~mL}$ 圆底瓶中, 加入约 $4 \mathrm{~mL}$ 无水 $\mathrm{CH}_{2} \mathrm{Cl}_{2}$ 溶剂, 室温条件下搅拌反应; $1 \mathrm{~h}$ 原料基本消失完全; 反 应液浓缩后, 湿法上样硅胶柱层析分离[洗脱剂: $V($ 二氯 甲烷 $): V($ 甲醇 $)=40: 1$ ]得产物点重约 $85 \mathrm{mg}$, 乙酸乙 酯重结晶得白色晶体 7 (85 mg, 94.4\%). m.p. 184 185 ${ }^{\circ} \mathrm{C} ;{ }^{1} \mathrm{H}$ NMR $\left(\mathrm{CDCl}_{3}, 400 \mathrm{~Hz}\right) \delta: 0.96 \sim 1.09(\mathrm{~m}, 9 \mathrm{H}, 3 \times$ $\mathrm{CH}_{3}$ in Camphanoyl), $1.36 \sim 1.38\left(\mathrm{~m}, 6 \mathrm{H}, 2 \times \mathrm{CH}_{3}\right.$ in isopropyl), $1.62 \sim 2.44\left(\mathrm{~m}, 4 \mathrm{H}, 2 \times \mathrm{CH}_{2}\right.$ in camphanoyl), $3.97\left(\mathrm{~s}, 3 \mathrm{H}, \mathrm{OCH}_{3}\right), 4.68 \sim 4.74(\mathrm{~m}, 1 \mathrm{H}, \mathrm{CH}$ in isopropyl), $5.43\left(\mathrm{~m}, 2 \mathrm{H}, \mathrm{CH}_{2} \mathrm{O}\right), 5.99$ (s, $\left.1 \mathrm{H}, 3-\mathrm{H}\right), 6.98$ (d, $J=8.78$ $\mathrm{Hz}, 1 \mathrm{H}, 6-\mathrm{H}), 8.16$ (d, $J=8.78 \mathrm{~Hz}, 1 \mathrm{H}, 5-\mathrm{H})$; MS (ESI) $m / z(\%): 461.2\left(\mathrm{M}^{+}+\mathrm{H}\right), 483.1\left(\mathrm{M}^{+}+\mathrm{Na}\right)$; HRMS calcd for $\mathrm{C}_{24} \mathrm{H}_{28} \mathrm{O}_{7} \mathrm{~S}\left(\mathrm{M}^{+}+\mathrm{H}\right) 461.1634$, found 461.1628 .

化合物 $\mathbf{1 4}$ 和 25: 化合物 $\mathbf{1 0 b}(1.88 \mathrm{~g}, 7.9 \mathrm{mmol})$ 加入 约 $10 \mathrm{~mL}$ 无水 $\mathrm{DMF}$ 溶解, 加入无水碳酸钾 $(4.36 \mathrm{~g}, 31.6$ $\mathrm{mmol})$, 滴加碘甲烷 $(1.7 \mathrm{~mL}, 27.65 \mathrm{mmol})$, 室温条件下 搅拌反应; $0.5 \mathrm{~h}$ 后原料反应完全, 反应液倾入 $2 \mathrm{~mol} \cdot \mathrm{L}^{-1}$ 盐酸 $\left(200 \mathrm{~mL}\right.$ )中, 乙酸乙酯萃取, 饱和 $\mathrm{Na}_{2} \mathrm{~S}_{2} \mathrm{O}_{3}$ 溶液洗, 饱和食盐水洗, 无水硫酸钠干燥, 过滤、浓缩后经硅胶 柱层析分离 [洗脱剂: $V($ 石油醚 $): V($ 乙酸乙酯 $)=6: 1$ ] 得白色固体 $14\left(570 \mathrm{mg}, 30.2 \%\right.$, m.p. $\left.100 \sim 102{ }^{\circ} \mathrm{C}\right)$ 和 $\mathbf{2 5}$ (850 mg, 45.3\%, m.p. $119 \sim 121{ }^{\circ} \mathrm{C}$ ).

1-硫杂-4-甲氧基-7-甲氧甲氧基- $2 H$-色烯-2-酮(14): ${ }^{1} \mathrm{H}$ NMR $\left(\mathrm{CDCl}_{3}, 400 \mathrm{~Hz}\right) \delta: 3.49$ (s, 3H, $\left.\mathrm{OCH}_{3}\right), 3.96$ (s, $\left.3 \mathrm{H}, 4-\mathrm{OCH}_{3}\right), 5.23\left(\mathrm{~s}, 2 \mathrm{H}, \mathrm{OCH}_{2} \mathrm{O}\right), 6.00(\mathrm{~s}, 1 \mathrm{H}, 3-\mathrm{H})$, 7.04 (dd, $J=8.99,2.37 \mathrm{~Hz}, 1 \mathrm{H}, 6-\mathrm{H}), 7.10(\mathrm{~d}, J=2.37 \mathrm{~Hz}$, $1 \mathrm{H}, 8-\mathrm{H}), 8.05$ (d, $J=8.99 \mathrm{~Hz}, 1 \mathrm{H}, 5-\mathrm{H})$; MS (EI) $\mathrm{m} / z$ (\%): $252\left(\mathrm{M}^{+}, 25.41\right), 224$ (18.58), 45 (100).

25: ${ }^{1} \mathrm{H}$ NMR $\left(\mathrm{CDCl}_{3}, 400 \mathrm{~Hz}\right) \delta$ : $1.51(\mathrm{~s}, 6 \mathrm{H}, 2 \times$ $\left.\mathrm{CH}_{3}\right), 3.50\left(\mathrm{~s}, 3 \mathrm{H}, \mathrm{OCH}_{3}\right), 5.24\left(\mathrm{~s}, 2 \mathrm{H}, \mathrm{OCH}_{2} \mathrm{O}\right), 6.90(\mathrm{~d}$, $J=2.33 \mathrm{~Hz}, 1 \mathrm{H}, 8-\mathrm{H}), 6.98(\mathrm{dd}, J=8.77,2.33 \mathrm{~Hz}, 1 \mathrm{H}$, 6-H), 8.03 (d, $J=8.77 \mathrm{~Hz}, 1 \mathrm{H}, 5-\mathrm{H}$ ); MS (EI) $\mathrm{m} / z$ (\%): 266 $\left(\mathrm{M}^{+}, 20.64\right), 196$ (24.01), 45 (100).

化合物 $\mathbf{2 6}$ 和 27: 化合物 $\mathbf{1 0 b}(1.88 \mathrm{~g}, 7.9 \mathrm{mmol})$ 加入 
约 $10 \mathrm{~mL}$ 无水 DMF 溶解, 加入无水碳酸钾 $(3.74 \mathrm{~g}, 26.86$ $\mathrm{mmol})$, 滴加溴乙烷 $(1.8 \mathrm{~mL}, 23.70 \mathrm{mmol})$, 室温条件下 摚拌反应; $1.5 \mathrm{~h}$ 后原料反应完全, 生成两个产物点; 反 应液倾入 $200 \mathrm{~mL}$ 冰水中, 析出白色固体, 硅胶柱层析 分离 [洗脱剂: $V$ (石油醚) $: V$ (乙酸乙酯 $)=6: 1$ ] 先后得白 色固体 26 (1.43 g, 75.6\%, m.p. 112 114 $\left.{ }^{\circ} \mathrm{C}\right)$ 和 27 (95 mg, 5\%, m.p. $\left.111 \sim 113{ }^{\circ} \mathrm{C}\right)$.

1-硫杂-4-乙氧基-7-甲氧甲氧基- $2 H$-色烯-2-酮(26): ${ }^{1} \mathrm{H}$ NMR $\left(\mathrm{CDCl}_{3}, 400 \mathrm{~Hz}\right) \delta: 1.52 \sim 1.54\left(\mathrm{~m}, 3 \mathrm{H}, \mathrm{CH}_{3}\right.$ in ethyl), 3.49 (s, $\left.3 \mathrm{H}, \mathrm{OCH}_{3}\right), 4.13 \sim 4.18\left(\mathrm{~m}, 2 \mathrm{H}, \mathrm{CH}_{2}\right.$ in ethyl), 5.24 (s, $\left.2 \mathrm{H}, \mathrm{OCH}_{2} \mathrm{O}\right), 5.98(\mathrm{~s}, 1 \mathrm{H}, 3-\mathrm{H}), 7.04$ (dd, $J=9.00,1.57 \mathrm{~Hz}, 1 \mathrm{H}, 6-\mathrm{H}), 7.09$ (d, $J=1.57 \mathrm{~Hz}, 1 \mathrm{H}, 8-\mathrm{H})$, 8.08 (d, $J=9.00 \mathrm{~Hz}, 1 \mathrm{H}, 5-\mathrm{H}$ ); MS (ESI) $m / z$ (\%): 267.1 $\left(\mathrm{M}^{+}+\mathrm{H}\right), 555.1\left(2 \mathrm{M}^{+}+\mathrm{Na}\right)$.

27: ${ }^{1} \mathrm{H}$ NMR $\left(\mathrm{CDCl}_{3}, 400 \mathrm{~Hz}\right) \delta: 1.48(\mathrm{t}, J=6.8 \mathrm{~Hz}$, $3 \mathrm{H}, \mathrm{CH}_{3}$ in ethyl), 3.50 (s, $\left.3 \mathrm{H}, \mathrm{OCH}_{3}\right), 4.22(\mathrm{q}, J=7.0 \mathrm{~Hz}$, $2 \mathrm{H}, \mathrm{CH}_{2}$ in ethyl), $5.25\left(\mathrm{~s}, 2 \mathrm{H}, \mathrm{OCH}_{2} \mathrm{O}\right), 6.30(\mathrm{~s}, 1 \mathrm{H}, 3-\mathrm{H})$, 7.11 (d, $J=2.35 \mathrm{~Hz}, 1 \mathrm{H}, 8-\mathrm{H}), 7.12$ (dd, $J=9.78,2.35 \mathrm{~Hz}$, 1H, 6-H), 8.38 (d, $J=9.78 \mathrm{~Hz}, 1 \mathrm{H}, 5-\mathrm{H}$ ); MS (ESI) $\mathrm{m} / \mathrm{z}$ (\%): $267.1\left(\mathrm{M}^{+}+\mathrm{H}\right), 555.1\left(2 \mathrm{M}^{+}+\mathrm{Na}\right)$.

化合物 28 和 29: 化合物 $\mathbf{1 0 b}(1.88 \mathrm{~g}, 7.9 \mathrm{mmol})$ 加入 约 $10 \mathrm{~mL}$ 无水 DMF 溶解, 加入无水碳酸钾 $(3.74 \mathrm{~g}, 26.86$ $\mathrm{mmol})$ 和溴代异丙烷 $(2.5 \mathrm{~mL}, 23.7 \mathrm{mmol})$, 加热至外温 $80{ }^{\circ} \mathrm{C}$ 搅拌反应, $3 \mathrm{~h}$ 后原料基本反应完, 反应液倾入 200 $\mathrm{mL}$ 冰水中, 析出白色固体, 硅胶柱层析分离[洗脱剂: $V($ 石油醚 $): V($ 乙酸乙酯 $)=6: 1$ ] 先后得白色固体化合 物 $28\left(1.62\right.$ g, $85.7 \%$, m.p. $\left.85 \sim 86{ }^{\circ} \mathrm{C}\right)$ 和 $\mathbf{2 9}(98 \mathrm{mg}, 5 \%$, m.p. $\left.84 \sim 86{ }^{\circ} \mathrm{C}\right)$.

1-硫杂-4-异丙氧基-7-甲氧甲氧基- $2 H$-色烯-2-酮 (28): ${ }^{1} \mathrm{H}$ NMR $\left(\mathrm{CDCl}_{3}, 500 \mathrm{~Hz}\right) \delta: 1.45 \sim 1.46(\mathrm{~m}, 6 \mathrm{H}, 2 \times$ $\mathrm{CH}_{3}$ in isopropyl), 3.49 (s, $\left.3 \mathrm{H}, \mathrm{OCH}_{3}\right), 4.68 \sim 4.73(\mathrm{~m}, 1 \mathrm{H}$, $\mathrm{CH}$ in isopropyl), $5.23\left(\mathrm{~s}, 2 \mathrm{H}, \mathrm{OCH}_{2} \mathrm{O}\right), 5.97(\mathrm{~s}, 1 \mathrm{H}, 3-\mathrm{H})$, 7.03 (dd, $J=8.99,2.37 \mathrm{~Hz}, 1 \mathrm{H}, 6-\mathrm{H}), 7.09$ (d, $J=2.37 \mathrm{~Hz}$, $1 \mathrm{H}, 8-\mathrm{H}), 8.06$ (d, $J=8.99 \mathrm{~Hz}, 1 \mathrm{H}, 5-\mathrm{H})$; MS (EI) $\mathrm{m} / \mathrm{z}(\%)$ : $280\left(\mathrm{M}^{+}, 29.60\right), 210$ (33.37), 45 (100).

29: ${ }^{1} \mathrm{H} \mathrm{NMR}\left(\mathrm{CDCl}_{3}, 500 \mathrm{~Hz}\right) \delta: 1.42 \sim 1.43(\mathrm{~m}, 6 \mathrm{H}$, $2 \times \mathrm{CH}_{3}$ in isopropyl), $3.50\left(\mathrm{~s}, 3 \mathrm{H}, \mathrm{OCH}_{3}\right), 4.77 \sim 4.80(\mathrm{~m}$, $1 \mathrm{H}, \mathrm{CH}$ in isopropyl), $5.24\left(\mathrm{~s}, 2 \mathrm{H}, \mathrm{OCH}_{2} \mathrm{O}\right), 6.31(\mathrm{~s}, 1 \mathrm{H}$, $3-\mathrm{H}), 7.10(\mathrm{~d}, J=2.35 \mathrm{~Hz}, 1 \mathrm{H}, 8-\mathrm{H}), 7.11$ (dd, $J=9.78$,
$2.35 \mathrm{~Hz}, 1 \mathrm{H}, 6-\mathrm{H}), 8.38$ (d, $J=9.78 \mathrm{~Hz}, 1 \mathrm{H}, 5-\mathrm{H})$; MS (ESI) $m / z(\%): 280\left(\mathrm{M}^{+}, 22.11\right), 238$ (14.59), 45 (100).

致谢 感谢本单位药物化学教研室付伟副研究员对化 合物 9a 和 $10 \mathrm{a}$ 利用高斯软件能量精确计算提供的帮助.

辅助材料(Supporting Information) 化合物 10b 的 $\mathrm{NOE}$ 和 HMBC 谱可以免费从本刊网站(http://siocjournal.cn/)上下载. CCDC-872263 \& 872264 含有本文 两个单晶结构的补充数据, 可在以下网址免费查询 www.ccdc.cam.ac. uk/data_request/cif.

\section{References}

[1] Lee, T. T.; Kashiwada, Y.; Huang, L.; Snider, J.; Cosentino, L. M.; Lee, K. H. Bioorg. Med. Chem., 1994, 2, 1051

[2] Xie, L.; Takeuchi, Y.; Cosentino, L. M.; Lee, K. H. Bioorg. Med. Chem. Lett. 1998, 8, 2151.

[3] Yu, D. L.; Chen, C. H.; Brossi, A.; Lee, K. H. J. Med. Chem. 2004, 47, 4072.

[4] Xia, P.; Yin, Z. J.; Chen, Y.; Zhang, Q.; Zhang, B. N.; Xia, Y.; Yang, Z. Y.; Kilgore, N.; Wild, C.; Morris-Natschke, S. L.; Lee, K. H. Bioorg. Med. Chem. Lett. 2004, 12, 3341.

[5] Tang, J.; Qian, K. D.; Zhang, B. N.; Chen, Y.; Xia, P.; Yu, D. L.; Xia, Y.; Yang, Z. Y.; Chen, C. H.; Morris-Natschke, S. L.; Lee, K. H. Bioorg. Med. Chem. 2010, 18, 4363.

[6] Chen, Y.; Cheng, M.; Liu, F. Q.; Xia, P.; Qian, K. D.; Yu, D. L.; Xia, Y.; Yang, Z. Y.; Chen, C. H.; Morris-Natschke, S. L.; Lee, K. H. Bioorg. Med. Chem. 2011, 46, 4924.

[7] Lau, C. K.; Belanger, P. C.; Dufresne, C.; Scheigetz, J. J. Org. Chem. 1987, 52, 1670.

[8] Frisch, M. J.; Trucks, G. W.; Schlegel, H. B.; Scuseria, G. E.; Robb, M. A.; Cheeseman, J. R.; Scalmani, G.; Barone, V.; Mennucci, B.; Petersson, G. A.; Nakatsuji, H.; Caricato, M.; Li, X.; Hratchian, H. P.; Izmaylov, A. F.; Bloino, J.; Zheng, G.; Sonnenberg, J. L.; Hada, M.; Ehara, M.; Toyota, K.; Fukuda, R.; Hasegawa, J.; Ishida, M.; Nakajima, T.; Honda, Y.; Kitao, O.; Nakai, H.; Vreven, T.; Montgomery, J. A.; Peralta, Jr., J. E.; Ogliaro, F.; Bearpark, M.; Heyd, J. J.; Brothers, E.; Kudin, K. N.; Staroverov, V. N.; Keith, T.; Kobayashi, R.; Normand, J.; Raghavachari, K.; Rendell, A.; Burant, J. C.; Iyengar, S. S.; Tomasi, J.; Cossi, M.; Rega, N.; Millam, J. M.; Klene, M.; Knox, J. E.; Cross, J. B.; Bakken, V.; Adamo, C.; Jaramillo, J.; Gomperts, R.; Stratmann, R. E.; Yazyev, O.; Austin, A. J.; Cammi, R.; Pomelli, C.; Ochterski, J. W.; Martin, R. L.; Morokuma, K.; Zakrzewski, V. G.; Voth, G. A.; Salvador, P.; Dannenberg, J. J.; Dapprich, S.; Daniels, A. D.; Farkas, O.; Foresman, J. B.; Ortiz, J. V.; Cioslowski, J.; Fox, D. J. Gaussian 09, Revision B.01, Gaussian, Inc., Wallingford CT, 2010.

(Li, L.; Fan, Y.) 